\title{
Cloning and Sequencing of Truncated Toxoplasma gondii Subtilisin-Like 1 Antigen
}

\author{
Ahmad Rouhizadeh, ${ }^{1}$ Ata A Ghadiri, ${ }^{2,}$ Mohammad Razi Jalali, ${ }^{1}$ Masoud Ghorbanpour, ${ }^{3}$ and \\ Mohammad Hossein Razi Jalali ${ }^{3}$ \\ ${ }^{1}$ Department of Clinical Sciences, Faculty of Veterinary Medicine, Shahid Chamran University of Ahvaz, Ahvaz, IR Iran \\ ${ }^{2}$ Cellular and Molecular Research Center and Department of Immunology, School of Medicine, Ahvaz Jundishapur University of Medical Sciences, Ahvaz, IR Iran \\ ${ }^{3}$ Department of Clinical Pathobiology, Faculty of Veterinary Medicine, Shahid Chamran University of Ahvaz, Ahvaz, IR Iran \\ "Corresponding author: Ata A Ghadiri, Cellular and Molecular Research Center and Department of Immunology, School of Medicine, Ahvaz Jundishapur University of Medical \\ Sciences, Ahvaz, IR Iran. E-mail: ata.ghadiri@hotmail.fr
}

Received 2014 October 12; Accepted 2014 October 12

\begin{abstract}
Background: Toxoplasma gondii is an obligate intracellular protozoan parasite which has significant medical and veterinary impact on all around the world. Tracking of specific antigens or antibodies for toxoplasmosis is the main choice in its diagnosis. Recombinant proteins will improve sensitivity and specificity and reduce problems of standardization and reproducibility of diagnostic kits. Toxoplasma gondii Subtilisin-like protein (TgSUB1) is a novel example of a glycosylphosphatidylinositol) GPI (-anchored protein which can be considered as a potential marker for serodiagnosis of toxoplasmosis.

Objectives: The aims of this study were to find out major antigenic parts of this whole protein and to develop a recombinant prokaryotic plasmid.

Methods: In this experimental study, using bioinformatics softwares Parker Hydrophilicity prediction and Bepipred linear Epitope prediction to select best highly antigenic region of this protein, a $744 \mathrm{bp}$ fragment was amplified by polymerase chain reaction (PCR) on cDNA obtained from $T$. gondii RNA. The PCR product was cloned in PCR2.1 vector and subcloned into expression pET28a vector. The PCR2.1-SUB1 and PET28a-SUB1constructs were analyzed by PCR, restriction analysis and sequencing.

Results: A highly antigenic region in the hydrophilic part of the protein including amino acid residues 549 to 795 was successfully cloned and the sequences were confirmed. All nucleotide sequences in the PCR product have 100\% homology with the published reference sequence.

Conclusions: Pairing bioinformatics tools and cloning of the candidate molecules in vaccine development studies and diagnostic approaches will have powerful impact on promotion of research in infectious diseases. This strategy is considered as available and inexpensive technology even in less developed countries where the infectious diseases like toxoplasmosis is prevalent.
\end{abstract}

Keywords: Cloning, Subtilisin-Like 1, Toxoplasma gondii

\section{Background}

Toxoplasma gondii (T. gondii) is an obligate intracellular protozoan parasite which causes toxoplasmosis in a wide range of hosts and results in high morbidity and mortality [1]. In human, Toxoplasmosis causes serious health problems for immunocompromised patients and in the fetus of the pregnant women primarily infected during pregnancy [2]. In animals, the disease makes economic losses by embryonic death and resorption, fetal death and mummification, abortion, stillbirth, and neonatal death. The parasite is mainly transmitted by food or water contaminated by sporulated oocysts in the faeces of infected felids as definitive hosts; by consumption of raw or undercooked meat containing the bradyzoite in tissue cysts and by transplacental transmission [3-5].

Most available commercial kits use native antigens pre- pared from tachyzoites grown in mice and/or cell culture of $T$. gondii. Increasing number of research on $T$. gondii recombinant antigens with publications showing their potential diagnostic value, might be introduce them as candidates versus the non-standardized whole-cell lysate of T. gondii antigens. Upgrading these tests with recombinant proteins will improve their sensitivity and specificity which will have major advantages for the diagnosis of toxoplasmosis and this will reduce the problems of antigen standardization and assay reproducibility which is a major problem of diagnostic laboratories worldwide [6]. The surface antigens SAG1 (p30) [7-11], SAG2 (P22) [7, 9, 10]; the dense granule antigens GRA1 (P24) [12-15], GRA2 (P28) [16, 17], GRA6 (P32) $[14,18,19]$ and GRA7 (P29) $[9,10,15,20,21]$ the rhoptry antigens ROP1 (P66) [17, 22, 23]; the microneme antigens MAG1 $[24,25]$ and MIC1 $[24,26]$ are among several antigens that have been used for detection of $T$. gondii-specific anti-

Copyright (c) 2016, Zahedan University of Medical Sciences. This is an open-access article distributed under the terms of the Creative Commons Attribution-NonCommercial 4.0 International License (http://creativecommons.org/licenses/by-nc/4.0/) which permits copy and redistribute the material just in noncommercial usages, provided the original work is properly cited. 
bodies in humans and animals.

Toxoplasma gondii Subtilisin-like protein 1 (TgSUB1) have been suggested to play an important role in survival of parasite. It is placed in the microneme organelle and is responsible in adhesion to the host during invasion. TgSUB1 is cotranslationally processed and undergoes additional proteolytic maturation after discharge from the micronemes and transport on the surface of parasites [27]. A glycosylphosphatidylinositol (GPI) anchor at the C terminal of the TgSUB1 protein allows its expression on the cell surface and probably mediates the attachment of the parasite to the host cell. TgSUB1 is first synthesized as a proprotein of $120 \mathrm{kDa}$ and mature protein equivalent to 90 $\mathrm{kDa}$. Upon stimulation of microneme secretion, secondary processing takes place that cleaves TgSUB1 to 82-and 70-kDa and 44-kDa forms [28].

\section{Objectives}

Taking into account the different post translation modification of this protein which may be, and their crucial role in parasite adhesion, we planned to identify major antigenic parts of this whole protein to be able to choose the most antigenic one and develop a recombinant plasmid using this part of antigen.

\section{Methods}

\subsection{Parasite}

BALB/c mice were infected intraperitoneally by $1 \times$ $10^{3}$ tachyzoites of $T$. gondii $\mathrm{RH}$ strain maintained in our animal facility laboratory of Ahvaz Jundishapur University of Medical Sciences. Ahvaz, Iran. After three to four days post infection peritoneal cavities of the animals were washed with $5 \mathrm{~mL}$ of cooled and sterile phosphate-buffered saline (PBS) containing streptomycin and penicillin and collected exudate was centrifuged at $50 \mathrm{xg}$ for 5 minutes to remove host cells. Then supernatant was washed and centrifuged twice at $1000 \mathrm{xg}$ for 10 minutes, and the tachyzoites were collected [3] and pelleted tachyzoites immediately dissolved in RLT buffer containing 2-ME (Qiagen, Germany) and kept at $-80^{\circ} \mathrm{C}$ until RNA extraction.

\subsection{Bioinformatics Analysis}

Toxoplasma gondii subtilisin-like protein (TgSUB1) mRNA, complete cds (NCBI GenBank accession no. AY043483.1) was analyzed using bioinformatics softwares Parker Hydrophilicity Prediction and also Bepipred linear epitope prediction to select best highly antigenic region of this protein. According to this analysis, forward primer (5'-CTGACAACTCACGCAGAAGGTC-3') and reverse primer
(5'- TTACACGGTCAGTCCAACAAC-3') have been designed by the program Primer3 and analyzed by the BLAST program of the National Center of Biotechnology Information (NCBI).

\subsection{RNA Extraction and cDNA Synthesis}

Total RNA was extracted by RNeasy Mini Kit (Qiagen, Germany) and RNA purity reflected by the 260/280 nm absorbance ratio and the concentration determined using a Nanodrop 1000 Spectrophotometer (Thermo Fisher scientific., USA). cDNA synthesis was done using RevertAid HMinus first-strand cDNA synthesis kit (Thermo Fisher Scientific., USA). Reverse transcription reaction was carried out in $20 \mu \mathrm{L}$ volume containing $0.5 \mu \mathrm{g}$ RNA, Oligo (dT) 18 primes, and the buffer and enzyme components according to the manufacturer's instructions.

\subsection{Polymerase Chain Reaction (PCR)}

The reaction of PCR consisted of $5 \mu \mathrm{L} 10$ X PCR buffer, $1.5 \mu \mathrm{L} \mathrm{MgCl}_{2}$ (50 mM), $1 \mu \mathrm{L}$ dNTPs mix (10 mM), $0.75 \mu \mathrm{L}$ (7.5 pmoles) of each primer, $0.75 \mu \mathrm{L}$ (7.5 unites) Super-Taq DNA polymerase (Genefanavan, Iran), $39 \mu \mathrm{L}$ distilled water and $0.75 \mu \mathrm{L}$ of template cDNA (total reaction volume $50 \mu \mathrm{L}$ ). PCR amplification was performed as follows: 1 cycle of $94^{\circ} \mathrm{C}$ for 2 minutes, 35 cycles of $94^{\circ} \mathrm{C}$ for 1 minute, $57^{\circ} \mathrm{C}$ for 1 minute, and $72^{\circ} \mathrm{C}$ for 90 seconds and followed by a final extension at $72^{\circ} \mathrm{C}$ for 10 minutes. To verify the result, $5 \mu \mathrm{L}$ of PCR product was electrophoresed in a $1 \%$ agarose gel, stained with safe stain. PCR product was purified using PCR purification kit (Qiagen, Germany). DNA purity and concentration were determined using the Nanodrop spectrophotometer.

\subsection{T/A Cloning}

\subsubsection{Ligation}

The TgSUB1 PCR product was cloned into PCR®2.1 vector via T/A cloning $®$ Kit (Invitrogen, USA) according to the manufacturer's instructions. Ligation reaction was performed with $1: 3$ vector to insert molar ratio and contained, $1 \mu \mathrm{L}$ of PCR product, $2 \mu \mathrm{L} 5 \mathrm{X}$ T4 DNA ligase reaction buffer, $2 \mu \mathrm{L}$ pCRß2.1 vector $(25 \mathrm{ng} / \mu \mathrm{L}), 4 \mu \mathrm{L}$ nuclease free distilled water and $1 \mu \mathrm{L}$ ExpressLinkTM T4 DNA Ligase (5 units) (Final volume $10 \mu \mathrm{L}$ ). After gentle mix and a brief centrifuge, the ligation reaction was incubated at room temperature for 15 minutes.

\subsubsection{Transformation}

For transformation recA, endAE. coli strain TOP10 (Invitrogen, USA) competent cells were prepared via $\mathrm{CaCl}_{2}$ method [29]. The $2 \mu \mathrm{L}$ of the ligation reaction was added to $100 \mu \mathrm{L}$ of competent cells in $1.5 \mathrm{~mL}$ vial. The vial was placed 
on ice for 30 minutes and incubated at $42^{\circ} \mathrm{C}$ for 90 seconds and then immediately placed on ice for 5 minutes. After that $900 \mu$ L S.O.C. antibiotic free medium added to transformed cells. Then the cells were incubated in a shaker incubator with $250 \mathrm{rpm}$ for 1 hour at $37^{\circ} \mathrm{C} .20$ and $100 \mu \mathrm{L}$ of transformation reaction were spread on LB agar plates containing $40 \mathrm{mg} / \mathrm{mL}$ X-gal for blue-white screening and 100 $\mathrm{mg} / \mathrm{mL}$ Ampicillin. The plates were incubated overnight at $37^{\circ} \mathrm{C}$ and then were transferred to $4^{\circ} \mathrm{C}$ for $2-3$ hours to allow for proper color development.

\subsection{Analysis of Recombinant Colonies and Plasmids}

\subsubsection{Colony PCR Analysis}

At least 10 white colonies (contained recombinant PCR2.1/SUB1 plasmids) and blue colonies (without recombinant plasmids) were picked and cultured on new LB + Amp agar plates for analysis by PCR and also plasmid isolation in order to restriction analysis and sequencing. Colonies on new plates were removed and boiled at $95^{\circ} \mathrm{C}$ for $5 \mathrm{~min}$ utes in $50 \mu \mathrm{L}$ sterile water and centrifuged. $10 \mu \mathrm{L}$ of supernatants were used as template for PCR reaction. PCR amplifications were done by forward and reverse specific TgSUB1 primers.

\subsubsection{Plasmid Isolation}

Plasmids were extracted using GF-1 plasmid DNA extraction kit (Vivantis, Malaysia) according to the manufacturer's instructions. Then $5 \mu \mathrm{L}$ of the extracted plasmids were analyzed by electrophoresis on $0.8 \%$ agarose gel stained with safe stain and size of products estimated with $1 \mathrm{~kb}$ DNA ladder. In order to restriction enzyme analysis the concentrations were determined using the Nanodrop spectrophotometer.

\subsubsection{Restriction Enzyme Analysis}

Plasmids were digested with a single BamHI restriction enzyme (recognition site is at upstream of MCS) and also double digested with BamHI and NotI (recognition site is at downstream of MCS) restriction enzymes that their sequences place in upstream and downstream vector sequence in $20 \mu \mathrm{L}$ volume contains: $1 \mu \mathrm{g}$ of plasmid, $2 \mu \mathrm{L}$ of RE $10 \mathrm{X}$ Buffer D, 0.2 $\mu$ L Acetylated BSA (10 $\mu \mathrm{g} / \mu \mathrm{L})$, sterile, deionized water and $1 \mu \mathrm{L}$ of each restriction enzyme $(10 \mathrm{u} / \mu \mathrm{L})$ (Promega, USA). The mixtures were incubated overnight at the optimum temperature $37^{\circ} \mathrm{C}$. Digested products were visualized on $0.8 \%$ agarose gel stained by safe stain and fragments size measured by using $1 \mathrm{~kb}$ molecular weight marker.

\subsubsection{Sequencing}

The recombinant pCR2.1/SUB1 plasmids were sequenced directionally by standard protocol with $\mathrm{ABI}$ 3730XL DNA Analyzer, (applied Biosystems, CA) using universal M13 reverse and forward primers in $\mathrm{PCR} 2.1$ vector. The results were analyzed and compared to database using the BLAST program (NCBI).

\subsection{Subcloning}

\subsubsection{Insert and Vector Preparation}

The gene fragment was sub-cloned into His-tagged fusion vector pET28a expression plasmid (Novagen, Germany). In order to sub-clone both plasmids digested by BamHI and NotI restriction enzymes in $50 \mu \mathrm{L}$ volume reaction: $3 \mu \mathrm{g}$ of plasmid, $4 \mu \mathrm{L}$ of RE $10 \mathrm{X}$ Buffer D, $0.4 \mu \mathrm{L}$ Acetylated BSA $(10 \mu \mathrm{g} / \mu \mathrm{L})$, sterile, deionized water and $1 \mu \mathrm{L}$ of each restriction enzyme $(10 \mathrm{u} / \mu \mathrm{L})$ (Promega, USA). The mixtures were incubated overnight at the optimum temperature $37^{\circ} \mathrm{C}$ then the enzymes inactivated by heating at $65^{\circ} \mathrm{C}$ for 15 minutes. DNA fragments were recovered from $0.8 \%$ agarose gel slice using QIAquick gel Extraction Kit (Qiagen, Germany) for further manipulations.

\subsubsection{Ligation and Transformation}

In order to ligate the digested vector PET28a with appropriate insert, a 1: 5 molar ratio vector/insert was used with T4 DNA ligase and incubate at room temperature for 30 minutes, then transformed into E. coli strain TOP 10 (Invitrogen, USA) competent cells as described for T/A cloning. Transformation reaction was dispensed on LB agar plates containing $50 \mathrm{mg} / \mathrm{mL}$ Kanamycin.

\subsubsection{Analysis of pET Recombinants}

The recombinant $\mathrm{pE} 28 \mathrm{a}$ expression vector containing TgSUB1 gene (pET28a/SUB1) was confirmed by colony PCR using TgSUB1 forward and reverse primers, Restriction analysis by BamHI and NotI restriction enzymes and sequencing using universal T7 terminator primers.

\section{Results}

Total RNA of RH strain of Toxoplasma gondii was extracted and converted to cDNA for use as template in PCR amplification of TgSUB1 gene. Bioinformatics tools permit us to predict the hydrophilic sites which were overlapped with most antigenic part of coding frame for TgSUB1 gene. Parker Hydrophilicity prediction and Bepipred linear epitope prediction analysis showed highly hydrophilic sites and highest antigenicity in C-terminal domain of the antigen. Bioinformatics analyses (Figures 1 and 2), showed a 
highly antigenic region in C-terminal domain of the antigen including amino acid residues 549 to 795 in cds (nucleotide sequence of 1735 to 2478). After PCR amplification, a 744 bp- fragment corresponding to the expected product of TgSUB1 cDNA has shown in gel electrophoresis (Figure $3 A$ ). Then, the purified PCR product was ligated into PCR®2.1 vector and pCR2.1/TgSUB1 recombinant plasmids were transformed into competent E. coli TOP10 cells. PCR amplifications of white colonies (contained recombinant pCR2.1/SUB1 plasmids) using forward and reverse SUB1 amplified fragments of $744 \mathrm{bp}$ (Figure 3B). The appropriate recombinant (pCR2.1/SUB1) and none-recombinant plasmids were compared on $0.8 \%$ agar gel electrophoresis (Figure 3C). Undigested, digested with BamHI and double digested with BamHI/NotI restriction enzymes recombinant pCR2.1/SUB1 plasmids were showed on $0.8 \%$ agarose gel electrophoresis (Figure 3D). Sequencing analysis of four recombinant plasmids showed that two of them had hundred-percent homology with RH strain SUB1 sequence ((NCBI GenBank accession no. AY043483.1) and there were not show any mutation in sequence of insert (TgSUB1 PCR product). PCR, restriction enzyme analysis and sequencing results showed that target gene was inserted correctly into the recombinant plasmid and reading frame verified.

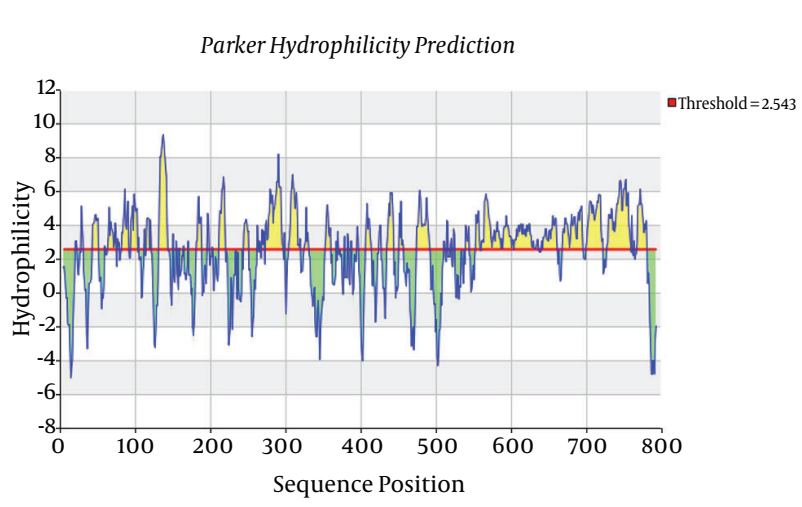

Figure 1. Hydrophilicity Index in C-Terminal Domain of TgSUB1 Showed by Parker Hydrophilicity Prediction Analysis

Both pCR2.1/SUB1 and pET28a expression plasmids were digested using same BamHI and NotI restriction enzymes and insert and vector fragments were recovered from $0.8 \%$ agarose gel slice gel extraction kit.

The recovered insert was ligated into linear expression vector and recombinant pET28a/SUB1 transformed into $E$. coli strain TOP10. The new recombinant plasmids were analyzed and confirmed by PCR, restriction analysis and sequencing (Figure 4).

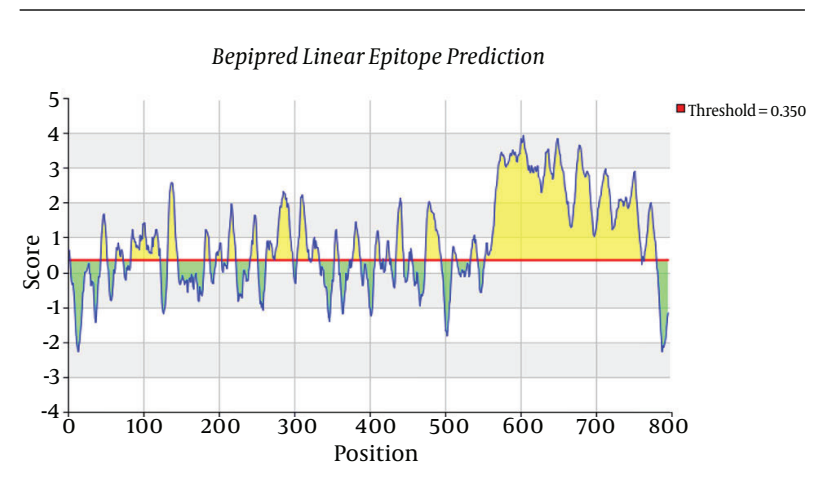

Figure 2. Highest Antigenicity Showed in the C-Terminal Domain of TgSUB1 by Bepipred Linear Epitope Prediction analysis

\section{Discussion}

In the present study, a highly antigenic region in the C-terminal domain of the TgSUB1 protein including amino acid residues 549 to 795 was recognized by bioinformatics analysis. Then a 744 bp PCR product was cloned in pCR2.1 vector and subcloned into expression pET28a vector and the recombinant plasmids were successfully transformed into E. coli TOP10 cells. PCR, restriction enzyme and sequencing analysis of the PCR2.1-SUB1 and PET28aSUB1constructs showed that target gene was inserted correctly into the recombinant plasmids and reading frame verified and also all nucleotide sequences in the PCR product have $100 \%$ homology with the published reference sequence.

Toxoplasma gondii has significant medical and veterinary impact all around the world [1]. There are several target genes of T. gondii which have been cloned and expressed into prokaryotic and eukaryotic expression systems. Surface antigens (SAGs), matrix antigens (MAGs), microneme proteins (MICs), rhoptry proteins (ROPs), and dense granule proteins (GRAs) are the most immunodominant and stage-specific antigens of $T$. gondii tachyzoite and bradyzoite stages [30]. In the present study the $744 \mathrm{bp} \mathrm{TgSUB1} \mathrm{PCR}$ product from $T$. gondii strain $\mathrm{RH}$ was cloned into pCRß2.1 vector via T/A Cloning® Kit (Invitrogen, USA) based on T/A cloning method. The $3.9 \mathrm{~kb}$ pCRß2.1 cloning vector is supplied in a linear form, ready-to-use for direct ligation of PCR product. This vector allows ampicillin and kanamycin selection, as well as blue/white colony screening. The vector contains several unique restriction endonuclease recognition sites around the cloning site, allowing easy restriction analysis of recombinant plasmids. The pET28 expression vectors encode an N-terminal His-tag/thrombin cleavage site/T7-tag sequence and an optional C-terminal His-Tag sequence. These vectors are used with lambda DE3 lysogen strains of E. coli. In these strains expression of a genomic 

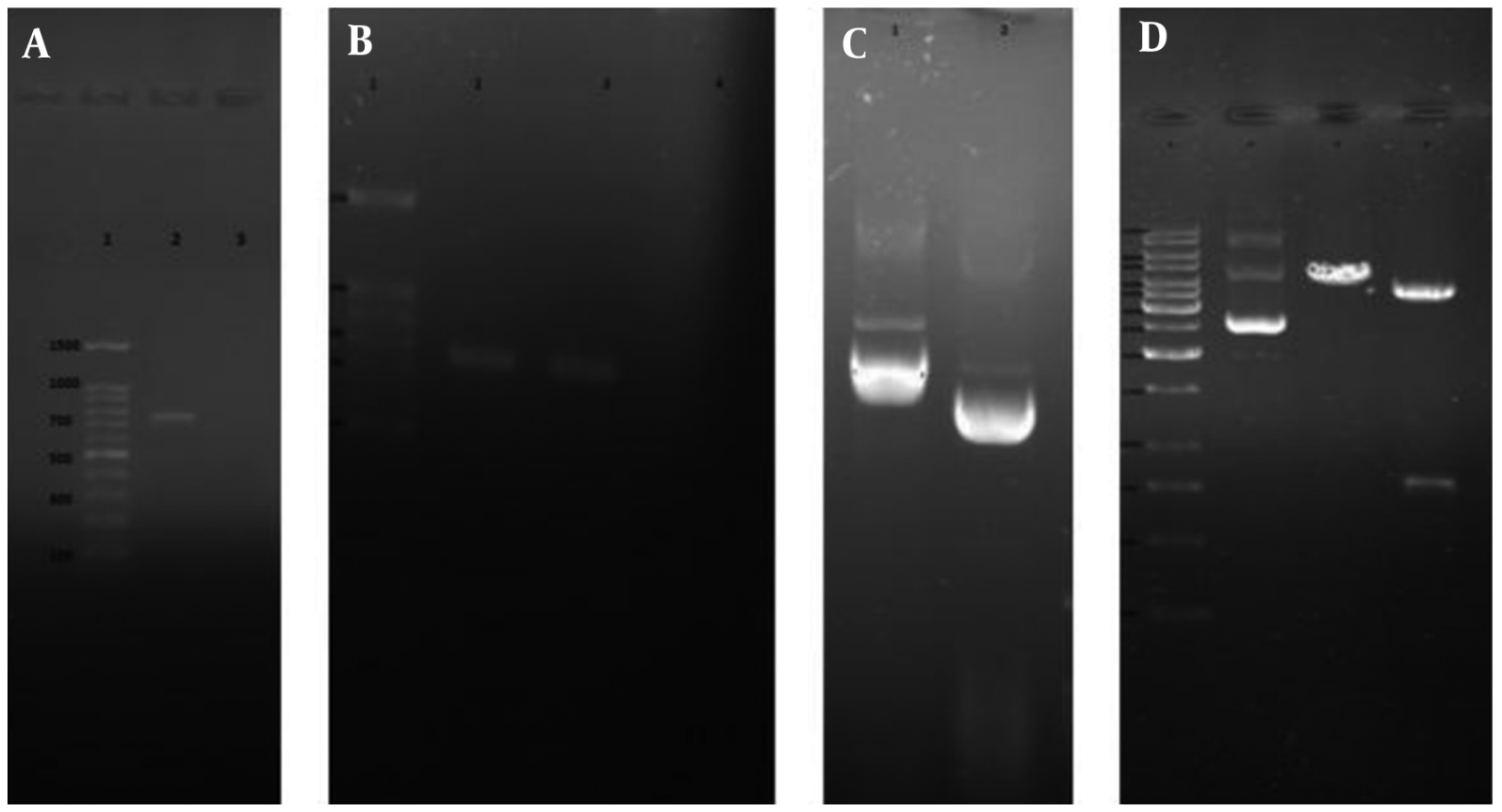

Figure 3. A, $1 \%$ agarose gel electrophoresis of TgSUB1 gene. Lane 1, 100 bp molecular weight marker; lane 2, 744 bp PCR product; B, colony PCR analysis of white and blue colonies by TgSUB1 Forward and Reverse primers. Lane 1,100bp molecular weight marker; lane 2, 744 bp PCR product of recombinant pCR2.1/SUB1 plasmid; lane 3, PCR product of non-recombinant pCR2.1/SUB1 plasmid (no band); C, extracted plasmids from white and blue colonies. Lane 1, recombinant plasmid (pCR2.1/SUB); lane 2, non-recombinant plasmid (pCR2.1); D, restriction analysis of pCR2.1/TgSUB1 plasmid. Lane 1, 1kb molecular weight marker; lane 2, undigested recombinant plasmid; lane 3, recombinant plasmid digested by BamHI; lane 3, recombinant plasmid digested by BamHI and NotI (shows released 818 bp fragment).

copy of the T7 RNA polymerase is under control of the lac repressor. The T7 promoter driven system for expression of heterologous proteins in E. coli is one of the most successful systems available because of its ability to stringently control the basal expression levels. Expression of the recombinant protein is induced by the addition of Isopropylb-D-thio-galactoside (IPTG) to the culture medium. Many recombinant antigens of T.gondii have been produced and some of them evaluated in serological test like ELISA and Western blot to identify different kinds of anti-T. gondii IgG, IgM, or IgA antibodies in attempts to produce appropriate diagnostic tools for the diagnosis of T. gondii infection and differentiation between the phases of toxoplasmosis [6].

Chahed Bel-Ochi et al. synthesized and evaluated a whole sequence of the recombinant $T$. gondii SAG1 antigen for screening of Toxoplasma IgG antibodies. They amplified PCR product and cloned it into the expression vector plasmid pET22b. They believed that truncated form of the SAG1 recombinant antigen might decrease its immunoreactivity, so whole SAG1 protein of the T. gondii RH strain was selected [8]. However, Velmurugan and colleagues evaluated diagnostic efficiency of truncated T. gondii SAG1 and GRA7 recombinant proteins, either individually or as a cocktail of them in goat sera by ELISA procedure. They used pGEM-T cloning vector and then sub-cloned them into pET-32b and pET-32c expression vectors, respectively and subsequently transformed into BL21 (DE3) pLysS cells. Expression of the histidine-tagged SAG1 and GRA7 fusion proteins yielded to high level of proteins and the recombinant proteins purified using Ni-NTA column [10]. Igarashi et al. cloned and expressed entire ROP2, GRA5 and GRA7 open reading frames of $T$. gondii $\mathrm{RH}$ strain into pET102/D-TOPO® vector which contains thioredoxin and polyhistidine tags at the $\mathrm{C}$ and $\mathrm{N}$ terminal ends and are expressed in $E$. coli BL21 (DE3) [20]. Mohabati et al. developed a recombinant ROP1 protein and assessed its antigenicity against human infected sera by Western blot assay. A1234 bp DNA fragment of ROP1gene from $T$. gondii RH strain was amplified by PCR cloned into pTZ57R/T vector and used pET-15b for its expression [23]. Eslamirad et al. cloned a 760 bp ROP1 gene from Toxoplasma gondii $\mathrm{RH}$ strain into pTZ57R vector and then sub-cloned it in eukaryotic expression vector, pcDNA3 [31]. In another study, Eslamirad et al. cloned an 1183bp ROP1 fragment into pTZ57R/T cloning vector and sub-cloned into pET32a expression plasmid [22].

Kotresha et al. also developed an IgG assay to identify antibodies against antigens which produced primarily during acute stage of toxoplasmosis using two recom- 

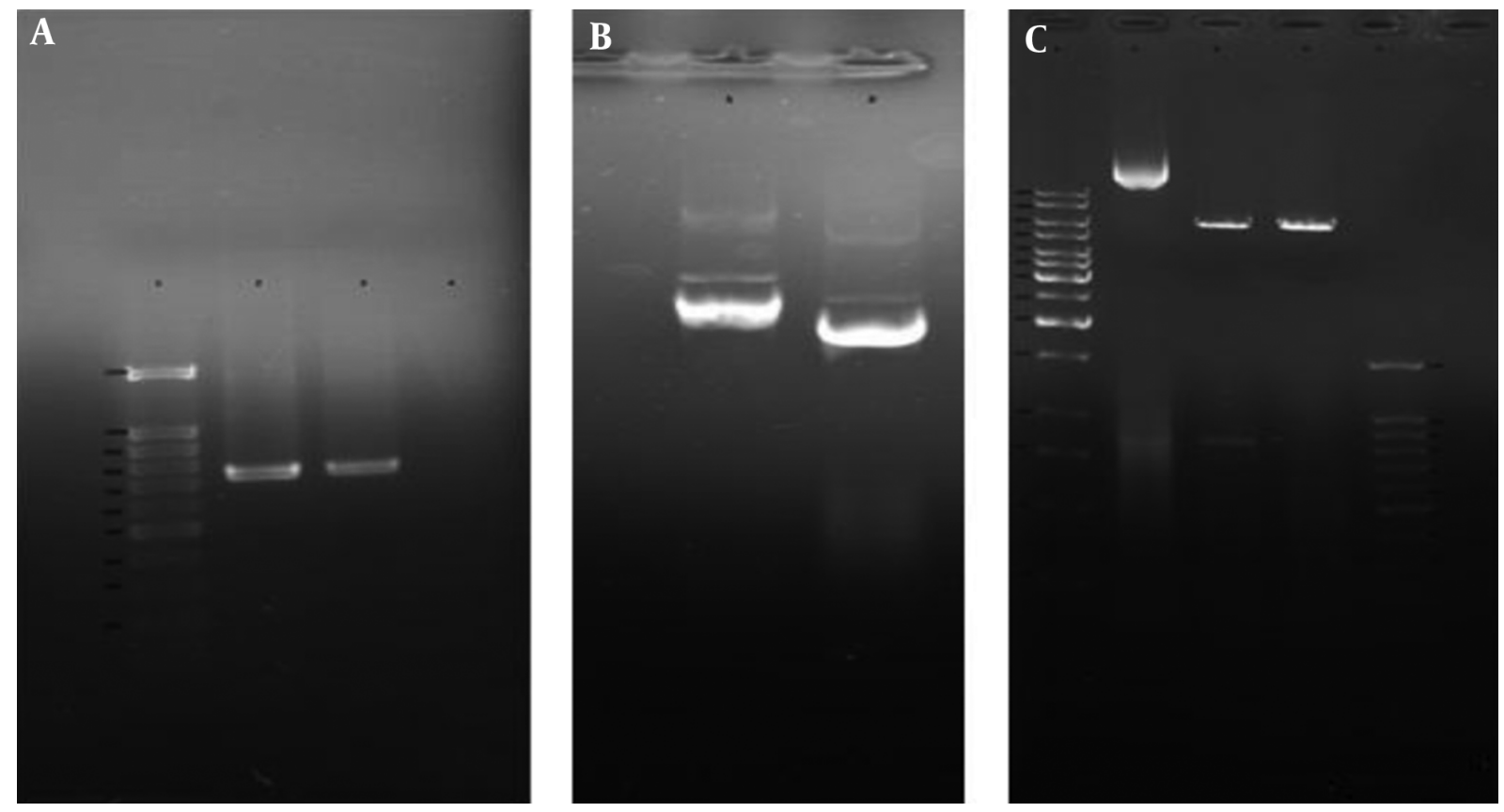

Figure 4. A, Colony PCR analysis of recombinant pET28a/SUB1 plasmids by TgSUB1 Forward and Reverse primers. Lane 1, 100 bp molecular weight marker; lanes 2 and 3, 744 bp PCR product of recombinant pET28a/SUB1 plasmids; lane 4, PCR product of non-recombinant pET28a/SUB1 plasmid (no band); B, Extracted recombinant and none-recombinant pET28a/SUB1 plasmids. Lane 1, recombinant plasmid (pET28a/SUB1); lane 2, non-recombinant plasmid (pET28a); C, Restriction analysis of pET28a/TgSUB1 plasmid. Lane 1, 1 kb molecular weight marker; lane 2, undigested recombinant plasmid; lane 3, recombinant plasmid digested by BamHI and NotI (shows released 818 bp fragment); lane 4 , recombinant plasmid digested by BamHI; lane 5: 100 bp molecular weight marker.

binant proteins of T. gondii GRA7 and SAG1 and evaluated diagnostic potential of these proteins. They used several bioinformatics softwares to identify highly antigenic regions of GRA7 and SAG1. They used TOPO cloning vector and then the fragments were subcloned into pProcEX HTa and pProcEX HTb expression vectors respectively [9]. Wang et al. cloned and expressed recombinant proteins GRA1 and GRA7 from T. gondii GT1 strain to use in serodiagnosis of toxoplasmosis in dogs by indirect ELISA. The GRA1 and GRA7 DNA fragments were amplified, and cloned into expression vector PET-28a to construct recombinant plasmids pET28GRA1 and pET28-GRA7, which were confirmed by restriction enzymes and sequencing, and were transformed into Escherichia coli BL21 (DE3). The immunoreactivity of the expressed proteins was confirmed by Western blot. The recombinant proteins GRA1 and GRA7 were purified using a Ni-NTA purification system, showing the purity of more than $95 \%$ [15]. Sadeghiani et al. developed recombinant GRA7 antigen and evaluated antigenicity of purified protein in immunoblot using human sera. They used genomic DNA from tachyzoites of $T$. gondii, RH strain for DNA extraction and amplified a $670 \mathrm{bp}$ fragment encoding amino acids 18 to 236 by PCR. They utilized PTZ57R/T as cloning vector and pET-28b as expression vector [21].
Selseleh et al. in two separate study evaluated usefulness of recombinant SAG1 and GRA7 for detection of $T$. gondii IgM and IgG antibodies against T. gondii in order to serodiagnosis of acute and chronic human toxoplasmosis by ELISA. SAG1 and GRA7 DNA fragments were amplified by PCR from T. gondii (RH Strain), were cloned into the PTZ57R cloning vector and ligation reactions were transformed in E. coli XL1-blue strain competent cells. Transformed cell were placed on agar plate containing X-gal and IPTG for blue/white screening of recombinant and nonrecombinant colonies. Restriction analysis of recombinant plasmids was done by BamH1 and Not1 restriction enzymes. The SAG1 and GRA7 fragments were sub-cloned in the pET-28a expression vector and transformed in E. coli Top10F. The plasmids with the correct inserts were confirmed by restriction analysis, PCR analysis and sequencing method [32, 33]. Subtilisin-like serine proteinases are kinds of MICs which have potential importance in Apicomplexan biology, as inhibitor. Studies have demonstrated that for successful invasion of host cells, serine proteinase activity is necessary [27]. TgSUB1 is a GPI-anchored micronemal protease that is first released onto the surface of parasites during invasion in a calcium-dependent manner and then shed into the media with other micronemal proteins. 
It is a novel example of a GPI-anchored protein in T. gondii that bypasses the GPI-dependent surface trafficking pathway to traffic to micronemes, specialized regulated secretory organelles $[27,28]$. Hruzik et al. recognized TgSUB1 as a potential marker for serodiagnosis of acute toxoplasmosis and used recombinant TgSUB1 in a line blot assay. They isolated RNA from tachyzoites of T. gondii strain NTE and used it for cDNA synthesis. The C-terminal fragment of the SUB1 gene (nucleotide positions 1657 to 2316) on the basis of the antigenic index containing amino acid residues 552 to 777 was prepared by PCR amplification and then 660-bp PCR product was cloned into $\mathrm{PQE}-30$ expression vector. Their results indicated that TgSUB1 in concert with other T. gondii recombinant antigens is suitable to improve diagnosis of T. gondii infection [34].

SUB1 is also studied in other genus of parasites, like Neospora caninum, It may be assumed that cross-reactions may occur with their closely related parasite $T$. gondii if such antigens applied in serological diagnosis. Ybanez et al. expressed two fragments of $N$. caninum containing five NcSUB1 tandem repeat copies coding for amino acids 524 to 843 (NcSUB1t) and 555 to 679 (NcSUB1tr). They demonstrated that NcSUB1 is highly conserved in N. caninum and have only $63 \%$ sequence identity to the T. gondii orthologue. Therefore, they demonstrated no cross-react with $T$. gondii in their ELISA method [35]. Hruzik et al. introduced Cterminal domain of TgSUB1 as a potential marker for serodiagnosis of acute toxoplasmosis. They suggested this recombinant protein can be used with other recombinant antigens such as SAG1 for diagnosis of infection [34].

Bioinformatics tools were the basic and major points in this study. By this way, the most antigenic parts of the whole protein are identified. Cloning and expression is more convenient than when a whole protein with high molecular weight. Purification and also folding of a 20 $30 \mathrm{kDa}$ recombinant protein is more practical than a 100 $\mathrm{kDa}$ or more molecular weight protein. We analyzed antigenic regions of $T$ gondii SUB1 by Parker Hydrophilicity prediction and also Bepipred Linear Epitope prediction programs to select best highly region of this protein. Selected region showed high Hydrophilicity at first program suggested that it contains surface epitopes. Beside, diagram of the second program demonstrated that this region has high antigenicity. These results are in agreement with studies showed a GPI anchor at the C terminal of the TgSUB1 which allow its expression on cell surface and probability mediates attachment of $T$. gondii to host cell. The sequencing results of pCR2.1/SUB1 recombinant plasmids showed 100\% homology of TgSUB1 PCR product with TgSUB1 sequence of NCBI GenBank accession no. AY043483.1 .Analysis of pET28a/SUB1 and pCR®2.1/SUB1 recombinant plasmids were showed their sequences are in-frame and there is not any mutation or deletion in the cloned fragment. We tried to develop truncated recombinant TgSUB1 antigen based on highly antigenic regions in C-terminal domain of TgSUB1 mRNA.

Gene cloning and expression in pET28a plasmid in $E$. coli cell as a prokaryotic system is an efficient method and also inexpensive which give large amounts of recombinant protein to do studies like antigenicity evaluations for development of vaccines and diagnostic kits. Further experiments are in development for expression and purification of this protein.

\section{Acknowledgments}

The authors would like to thank the staff of the cell and molecular research center, Ahvaz Jundishapur University of Medical Sciences, Ahvaz, Iran. The authors wish to thank from the Shahid Chamran University of Ahvaz for support through grants no 93/177770.

\section{Footnotes}

Authors' Contribution: The work presented here was carried out in collaboration between all authors: All authors contributed equally to the design and performance of the study. They participated in the laboratory evaluation and performed the literature review, drafted the manuscript.

Conflict of Interest: The authors declare that there is no conflict of interests regarding the publication of this paper.

Funding/Support: Shahid Chamran University of Ahvaz.

\section{References}

1. Pal M, Alem B, Gar G, Tuli G. Toxoplasmosis in animals and humans, Its diagnosis, epidemiology and control. Int J Livest Res. 2014;4(2):1-10.

2. Montoya JG, Liesenfeld O. Toxoplasmosis. Lancet. 2004;363(9425):1965-76. doi: 10.1016/S0140-6736(04)16412-X. [PubMed: 15194258].

3. Canan K, Guuml lay AA, Ccedil igdem GNR, Kerem Y, Ali MA. The role of various proteins of Toxoplasma gondii in provoking immune response at different stages of infection. Afr J Microbiol Res. 2013;7(2):98102. doi: 10.5897/ajmr12.896.

4. Dubey JP. Strategies to reduce transmission of Toxoplasma gondii to animals and humans. Vet Parasitol. 1996;64(1-2):65-70. [PubMed: 8893464].

5. Dubey JP, Lindsay DS. Neosporosis, toxoplasmosis, and sarcocystosis in ruminants. Vet Clin North Am Food Anim Pract. 2006;22(3):645-71. doi: 10.1016/j.cvfa.2006.08.001. [PubMed: 17071358].

6. Holec-Gasior L. Toxoplasma gondii recombinant antigens as tools for serodiagnosis of human toxoplasmosis: current status of studies. Clin Vaccine Immunol. 2013;20(9):1343-51. doi: 10.1128/CVI.00117-13. [PubMed: 23784855]. 
7. Khanaliha K, Motazedian MH, Kazemi B, Shahriari B, Bandehpour M, Sharifniya Z. Evaluation of recombinant SAG1, SAG2, and SAG3 antigens for serodiagnosis of toxoplasmosis. Korean J Parasitol. 2014;52(2):137-42. doi: 10.3347/kjp.2014.52.2.137. [PubMed: 24850956].

8. Chahed Bel-Ochi N, Bouratbine A, Mousli M. Enzyme-linked immunosorbent assay using recombinant SAG1 antigen to detect Toxoplasma gondii-specific immunoglobulin $\mathrm{G}$ antibodies in human sera and saliva. Clin Vaccine Immunol. 2013;20(4):468-73. doi: 10.1128/CVI.00512-12. [PubMed: 23345586].

9. Kotresha D, Poonam D, Muhammad Hafiznur Y, Saadatnia G, Nurulhasanah O, Sabariah O, et al. Recombinant proteins from new constructs of SAG1 and GRA7 sequences and their usefulness to detect acute toxoplasmosis. Trop Biomed. 2012;29(1):129-37. [PubMed: 22543613].

10. Velmurugan GV, Tewari AK, Rao JR, Baidya S, Kumar MU, Mishra AK. High-level expression of SAG1 and GRA7 gene of Toxoplasma gondii (Izatnagar isolate) and their application in serodiagnosis of goat toxoplasmosis. Vet Parasitol. 2008;154(3-4):185-92. doi: 10.1016/j.vetpar.2008.03.032. [PubMed: 18495348].

11. Wang Y, Wang G, Zhang D, Yin H, Wang M. Screening and identification of novel B cell epitopes of Toxoplasma gondii SAG1. Parasit Vectors. 2013;6:125. doi: 10.1186/1756-3305-6-125. [PubMed: 23631709].

12. Hiszczynska-Sawicka E, Brillowska-Dabrowska A, Dabrowski S, Pietkiewicz H, Myjak P, Kur J. High yield expression and single-step purification of Toxoplasma gondii SAG1, GRA1, and GRA7 antigens in Escherichia coli. Protein Expr Purif. 2003;27(1):150-7. [PubMed: 12509997].

13. Beghetto E, Pucci A, Minenkova O, Spadoni A, Bruno L, Buffolano W, et al. Identification of a human immunodominant B-cell epitope within the GRA1 antigen of Toxoplasma gondii by phage display of cDNA libraries. Int J Parasitol. 2001;31(14):1659-68. [PubMed: 11730793]

14. Lecordier L, Fourmaux MP, Mercier C, Dehecq E, Masy E, CesbronDelauw MF. Enzyme-linked immunosorbent assays using the recombinant dense granule antigens GRA6 and GRA1 of Toxoplasma gondii for detection of immunoglobulin G antibodies. Clin Diagn Lab Immunol. 2000;7(4):607-11. [PubMed: 10882660].

15. Wang Z, Ge W, Huang SY, Li J, Zhu XQ, Liu Q. Evaluation of recombinant granule antigens GRA1 and GRA7 for serodiagnosis of Toxoplasma gondii infection in dogs. BMC Vet Res. 2014;10:158. doi: 10.1186/17466148-10-158. [PubMed: 25016474].

16. Golkar M, Rafati S, Abdel-Latif MS, Brenier-Pinchart MP, FrickerHidalgo $\mathrm{H}$, Sima $\mathrm{BK}$, et al. The dense granule protein GRA2, a new marker for the serodiagnosis of acute Toxoplasma infection: comparison of sera collected in both France and Iran from pregnant women. Diagn Microbiol Infect Dis. 2007;58(4):419-26. doi: 10.1016/j.diagmicrobio.2007.03.003. [PubMed: 17509806].

17. Holec-Gasior L, Kur J, Hiszczynska-Sawicka E. GRA2 and ROP1 recombinant antigens as potential markers for detection of Toxoplasma gondii-specific immunoglobulin $\mathrm{G}$ in humans with acute toxoplasmosis. Clin Vaccine Immunol. 2009;16(4):510-4. doi: 10.1128/CVI.0034108. [PubMed: 19225074 ]

18. Golkar M, Azadmanesh K, Khalili G, Khoshkholgh-Sima B, Babaie $\mathrm{J}$, Mercier C, et al. Serodiagnosis of recently acquired Toxoplasma gondii infection in pregnant women using enzymelinked immunosorbent assays with a recombinant dense granule GRA6 protein. Diagn Microbiol Infect Dis. 2008;61(1):31-9. doi: 10.1016/j.diagmicrobio.2007.09.003. [PubMed: 18249081].

19. Myjak P. Efficient production of the Toxoplasma gondii GRA6, p35 and SAG2 recombinant antigens and their applications in the serodiagnosis of toxoplasmosis. Acta Parasitol. 2005;50(3):249-54.

20. Igarashi M, Kano F, Tamekuni K, Kawasaki PM, Navarro IT, Vidotto $\mathrm{O}$, et al. Toxoplasma gondii: cloning, sequencing, expression, and antigenic characterization of ROP2, GRA5 and GRA7. Genet Mol Res. 2008;7(2):305-13. [PubMed: 18551396].

21. Sadeghiani G, Zare M, Babaie J, Shokrgozar MA, Azadmanesh K, FardEsfahani P, et al. Heterologous production of dense granule GRA7 antigen of Toxoplasma gondii in Escherichia coli. Southeast Asian J Trop Med Public Health. 2009;40(4):692-700. [PubMed: 19842401].

22. Eslamirad Z, Ghorbanzadeh B, Haji Hosein R, Abtahi H, Mosayebi M, Shojaee S, et al. Cloning of 1183 bp Fragment from Rhoptry Protein I (ROPI) Gene of Toxoplasma gondii (RH) in Expression Prokaryote Plasmid PET32a. Zahedan J Res Med Sci. 2013;15(10):32-6.

23. Mohabati R, Babaie J, Amiri S, Talebzadeh M, Fard-Esfahani P, Darbouy $\mathrm{M}$, et al. Expression and Purification of Recombinant ROP1 of Toxoplasma gondii in Bacteria. Avicenna J Med Biotechnol. 2013;5(4):227-33. [PubMed: 24285997].

24. Holec-Gasior L, Ferra B, Drapala D, Lautenbach D, Kur J. A new MIC1MAG1 recombinant chimeric antigen can be used instead of the Toxoplasma gondii lysate antigen in serodiagnosis of human toxoplasmosis. Clin Vaccine Immunol. 2012;19(1):57-63. doi:10.1128/CVI.05433-11. [PubMed: 22116686].

25. Holec L, Hiszczynska-Sawicka E, Gasior A, Brillowska-Dabrowska A Kur J. Use of MAG1 recombinant antigen for diagnosis of Toxoplasma gondii infection in humans. Clin Vaccine Immunol. 2007;14(3):220-5. doi: 10.1128/CVI.00419-06. [PubMed: 17202305].

26. Holec L, Gasior A, Brillowska-Dabrowska A, Kur J. Toxoplasma gondii: enzyme-linked immunosorbent assay using different fragments of recombinant microneme protein 1 (MIC1) for detection of immunoglobulin G antibodies. Exp Parasitol. 2008;119(1):1-6. doi: 10.1016/j.exppara.2007.12.002. [PubMed: 18207143].

27. Binder EM, Lagal V, Kim K. The prodomain of Toxoplasma gondii GPIanchored subtilase TgSUB1 mediates its targeting to micronemes. Traffic. 2008;9(9):1485-96. doi: 10.1111/j.1600-0854.2008.00774.x. [PubMed: 18532988].

28. Miller SA, Binder EM, Blackman MJ, Carruthers VB, Kim K. A conserved subtilisin-like protein TgSUB1 in microneme organelles of Toxoplasma gondii. J Biol Chem. 2001;276(48):45341-8. doi: 10.1074/jbc.M106665200. [PubMed: 11564738].

29. Sambrook J, Fritsch EF, Maniatis T. Molecular cloning a laboratory manual. 2nd ed. NewYork: Cold spring harbor laboratory press; 1989.

30. Kotresha D, Noordin R. Recombinant proteins in the diagnosis of toxoplasmosis. APMIS. 2010;118(8):529-42. doi: 10.1111/j.16000463.2010.02629.x. [PubMed: 20666734].

31. Eslamirad Z, Dalimi A, Ghaffarifar F, Sharifi Z. Cloning rhoptry protein 1(RoP1) gene of Toxoplasma gondii (RH) in expression vector. Arch Razi Inst. 2008;63(2):11-7. 64.

32. Selseleh M, Keshavarz H, Mohebali M, Shojaee S, Selseleh M, Eshragian MR, et al. Production and evaluation of Toxoplasma gondii recombinant GRA7 for serodiagnosis of human infections. Korean J Parasitol. 2012;50(3):233-8. doi: 10.3347/kjp.2012.50.3.233. [PubMed: 22949752].

33. Selseleh MM, Keshavarz H, Mohebali M, Shojaee S, Modarressi M, Eshragian M, et al. Production and Evaluation of Toxoplasma gondii Recombinant Surface Antigen 1 (SAG1) for Serodiagnosis of Acute and Chronic Toxoplasma Infection in Human Sera. Iran J Parasitol. 2012;7(3):1-9. [PubMed: 23109955].

34. Hruzik A, Asif AR, Gross U. Identification of Toxoplasma gondil SUB1 antigen as a marker for acute infection by use of an innovative evaluation method. J Clin Microbiol. 2011;49(7):2419-25. doi: 10.1128/JCM.00464-11. [PubMed: 21543561].

35. Ybanez RH, Terkawi MA, Kameyama K, Xuan X, Nishikawa Y. Identification of a highly antigenic region of subtilisin-like serine protease 1 for serodiagnosis of Neospora caninum infection. Clin Vaccine Immunol 2013;20(10):1617-22. doi: 10.1128/CVI.00352-13. [PubMed: 23966554]. 